\title{
Review of Multi-criteria Optimization Methods - Theory and Applications
}

\author{
G. O. Odu, and O. E. Charles-Owaba \\ ${ }^{a}$ Faculty of Engineering, Delta State University, Abraka, Oleh Campus, Nigeria \\ ${ }^{b}$ Faculty of Technology, University of Ibadan, Ibadan, Nigeria
}

\begin{abstract}
A review of multi-criteria optimization concepts and methods is presented. The techniques provide solutions to the problems involving conflicting and multiple objectives. Several methods based on weighted averages, priority setting, outranking and their combinations are employed. The application and procedure in respect to material selection is presented. It is observed from the literature that no single approach is superior, rather, the selection of a specific method depends on the type of information that is provided in the problem, the designer's preferences, the solution requirements and the availability of software adopted.
\end{abstract}

Keywords: - Decision making, multi-criteria, multi-objective optimization, preferences, material selection,

\section{INTRODUCTION}

Optimization is an engineering discipline where extreme values of design criteria are sought. However, quite often there are multiple conflicting criteria that need to be handled. Satisfying one of these criteria comes at the expense of another. Multi-objective optimization deals with such conflicting objectives. It provides a mathematical framework to arrive at optimal design state which accommodates the various criteria demanded by the application. The process of optimizing systematically and simultaneously a collection of objective functions are called multi-objective optimization (MOO).

The similarity between single- and multi-objective optimization makes it possible to use the same optimization algorithms as for the single-objective case. The only required modification is to transform the multi-objective problem into a single criterion optimization problem. But the resulting solution to the single objective optimization problem is usually subjective to the parameter settings chosen by the user (Stadler, 1984; Mohammad, 2005). Moreover, multi-criteria optimization requires simultaneous optimization of multiple often competing or conflicting criteria (of objectives).

A multiple criteria decision making (MCDM) process is a system that helps with making decisions under multiple, but conflicting criteria. The conflict of these objectives or criteria arises because improvement in one objective can only be made to the detriment of one or more of the other objectives. It can also be described as a migrated system with an analyzing technique called multi-criteria decision analysis (MCDA). MCDA provides a systematic procedure to help decision makers choose the most desirable and satisfactory alternative under uncertain situations (Steven, 2000). For instance, in order to select a new automobile vehicle, many criteria need to be considered including the cost, speed, interior capacity, comfort level, and reliability. There is no optimal solution for this vehicle selection problem. One might want to choose a fast but inexpensive vehicle, while others might want a comfortable and reliable one. Therefore, using MCDA, a decision can be made according to the decision maker's preference.

A multiple-criteria problem begins when a decision maker has a situation that requires a decision. There are a number of criteria that the decision maker should be concerned with, and several different courses of action may be available to address most or all of the criteria in some way. The problem the decision maker is faced with is to determine which course of action or alternative would best satisfy the criteria and fully satisfy the constraints (Rao and Davin, 2008). According to many authors (see for instance, Zimmermann, 1991) MCDM is divided into Multi-objective Decision Making (MODM) and Multi-attribute Decision Making (MADM).

MODM studies decision problems in which the decision space is continuous. A typical example is mathematical programming problems with multiple objective functions. On the other hand, MADM concentrates on problems with discrete decision spaces. In these problems the set of decision alternatives has been predetermined.

Alternatives represent the different choices of action available to the decision maker. Usually, the set of alternatives is assumed to be finite, ranging from several to hundreds. They are supposed to be screened, prioritized and eventually ranked. Each MADM problem is associated with multiple attributes. Attributes are also referred to as "goals" or "decision criteria". Attributes represent the different dimensions from which the alternatives can be viewed. 
Almost all methodologies within multi-criteria decision analysis (MCDA) share similar steps of organization and decision matrix construction, but each methodology synthesizes information differently (Yoe, 2002; Fiqueira et al 2005). Different methods require diverse types of value information and follow various optimization algorithms. Some techniques rank options, some identify a single optimal alternative, some provide an incomplete ranking, and others differentiate between acceptable and unacceptable alternatives.

This paper therefore review the different multi-criteria optimization methods and its applications.

\section{OVERVIEW OF MULTI-CRITERIA DECISION MAKING (MCDM) METHODS}

Much of the early pioneering work with multi-objective optimization focuses specifically on structural design (Koski 1979, 1980).

There is no formal study of when decision making began (Koksalan et al. 2013). It is possible to trace the origin of decision analysis/utility theory and multiple objective mathematical programming. The roots of modern decision analysis/utility theory was noticed in the early work on the indifference contours by Edgeworth in 1880s, and was developed by Ramsey and de Finetti in the early 1930s and von Neumann and Morgenstein in 1940s. The developments of multiple objective mathematical programming were rather independent of decision analysis/utility theory. Often the demarcating feature between decision analysis/utility theory and multiple objective mathematical programming was that the formal addressed problems under uncertainty, the latter deterministic problems. The main drive to the development of multiple objective mathematical programming was provided by linear programming and goal programming in particular. Multiple objective mathematical programming was developed mainly during the 1970s (Koksalan et al. 2013).

Multi-objective optimization originally grew out of three areas: economic equilibrium and welfare theories, game theory, and pure mathematics (Marler and Arora 2004). According to Koksalan et al. (2013), the first known recorded work on multi-criteria decision making (MCDM) was carried out by the famous American statesman Benjamin Franklin. Even before Franklin's times, Aristotle (348-322 BC), a famous Greek philosopher and polymath, defines 'preferences' as 'rational desires'. This might have been the first time where someone made the connection between rational decision making and human desires (preferences). Another researcher who has contributed immensely to the development of early MCDM approach was Vilfredo Pareto (1848-1923), the French-Italian economist, probably the first researcher whose work might formally be classified as MCDM who established an optimality concept in the field of economics based on multiple objectives. A Pareto front is generated that allows designers to trade-off one objective against another (Mohammad, 2005). In his work (1906), he made the famous observation that $20 \%$ of the population of Italy owned $80 \%$ of the property. This was later interpreted by Joseph M. Juran as the Pareto principle (also termed the 80-20 rule). Pareto was the first (or at least one of the first) to mathematically study the aggregation of confronting criteria into a single composite index. He was also the first to introduce the concept of efficiency (which became known as Pareto-optimality), one of the key concepts of economics, negotiation science and modern MCDM theory.

Although various methods of multi-criteria optimization are common, they are often incomplete in terms of comprehensive coverage, algorithm presentation, and general applicability to engineering design. For instance, some authors only focuses on specific applications (Shanian and Savadogo 2006a, 2009; Chi-Nung Liao et al. 2011; Ashby 2000).

Multi-objective optimization in material design and selection was proposed and illustrated by Ashby (2000) where he developed a performance metrics characterizing each of the objective functions. Ashby (2005) developed material selection chart for a wide range of materials. The material selection procedure is performed based on two performance indices per chart. However, the graphical method is limited to, at most two objectives.

Marler and Arora (2009) proposed new insights into characteristics of the weighted-sum method with far reaching implications concerning the conceptual significance of the weights and techniques for maximizing the effectiveness of the method with respect to a priori articulation of preferences. Deb (2001) suggested a multi-objective genetic algorithm (GA) to solve the goal programming problem, where each goal were converted into an equivalent objective function, unlike the weighted goal programming method does not add any artificial constraint into its formulation. In addition, Romero (1985) shows that virtually all the multiobjective and goal programming approaches can be thought of as special cases of a general distance function model.

Applications of the multi-objective optimization (MOP) approach in agricultural planning were seen in the work of Romero et al. (1987) where the compromise programming was adopted. Elahi et al. (2011) proposed fuzzy compromise programming solution for supplier selection in quantity discounts situation. Also, Mohammad (2005) applied the multi-criteria optimization method in surface location error and material removal rate in high speed milling under uncertainty. 
In the work of Linkov et al. (2004), maxi-min method was discussed, based upon a strategy that tries to avoid the worst possible performance, maximizing the minimal performing criterion. The alternative for which the score of its weakest criterion is the highest is preferred. The maxi-min method can be used only when all criteria are comparable so that they can be measure on a common scale, which is a limitation.

Messac and Hattis (1996) apply physical programming to the design of high-speed transport planes. Messac and Wilson (1998) apply physical programming to the design of a robust controller for a two degree-of-freedom spring-and-mass system. Physical programming has also been used with complex problems such as finite element sizing optimization involving inflatable thin-walled structural members for housing.

In the work of Shanian and Savadogo (2009), the Technique of ranking Preferences by Similarity to the Ideal Solution (TOPSIS) in a deterministic domain was used. It is believed that the method has good potential for solving the material selection problem of fuel cell components like bipolar plates.

Rao and Patel (2011) proposed a novel multiple attribute decision making (MADM) method for solving the material selection problem. The method considers the objective weights of importance of the attributes as well as the subjective preferences of the decision maker to decide the integrated weights of importance of the attributes.

\subsection{Multi-criteria Decision making (MCDM) Methods}

In literature, multi-criteria decision making (MCDM) methods and optimization approaches have been applied in different ways. The problem with MCDM methods are generally a selection problem whereby one attempts to select the best or optimal alternative from a pre-specified but finite set of alternative. Selecting a particular MADM method depends on the characteristics of a problem, and is also partly based on the decision maker's preference. To solve MADM problems with homogenous data type, two approaches can be used. Firstly, data can be treated exclusively in order to form a set of uniform input parameters, and classical MADM methods can be used to solve the problem. Secondly, MADM methods should be modified in order to accept mixed input parameters. Both approaches should lead to the same result, but the former one seems simpler and more efficient (Hwang et al. 1992). In recent literature, common MADM methods found include Simple Weighted Addition method (SWA), Analytical Hierarchical Process (AHP), and different outranking methods such as the Elimination and Choice Translating Reality (ELECTRE).

The AHP was developed by Saaty (Saaty 1980, 1992). The essence of the process is decomposition of a complex problem into a hierarchy with goal (objective) at the top of the hierarchy, criterions, and subcriterions at levels and sub-levels of the hierarchy, and decision alternatives at the bottom of the hierarchy. In the standard AHP model the decision maker judgments are organized into pairwise comparison matrices at each level of the hierarchy. The judgments are point's estimates of the preference between two elements of the level. Let us denote the paired comparison matrix $A=\left\{a_{i j} \mid a_{j i}=1 / a_{i j}, a_{i j}>0, i, j=1,2, . k\right\}$, where k is the number of elements of the particular level. Saaty (1990) proposes to use preference expression $a_{i j}$ integers in the range 1 through 9, where 1 means that the $i$-th and the $j$-th element are equally important and 3 moderately more, 5 strongly more, 7 very strongly and 9 means that $i$-th element is absolutely more important than the $j$-th element. The values $2,4,6$, and 8 are allotted to indicate comparison values of importance. Ratio scale and the use of verbal comparisons are used for weighting of quantifiable and non-quantifiable elements. The method computes and aggregates their eigenvectors until the composite final vector of weight coefficients for alternatives is obtained. The entries of final weight coefficients vector reflect the relative importance (value) of each alternative with respect to the goal stated at the top of hierarchy. To elicit pair wise comparisons performed at a given level, a matrix $\mathrm{A}$ is created in turn by putting the result of pair wise comparison of element $i$ with element $j$ into the position $a_{i j}$ as below:

$$
A=\left[\begin{array}{llll}
a_{11} & a_{12} & \ldots & a_{1 n} \\
a_{21} & a_{22} & \ldots & a_{2 n} \\
a_{n 1} & a_{n 2} & \ldots & a_{n n}
\end{array}\right]
$$

After obtaining the weight vector, it is then multiplied with the weight coefficient of the element at a higher level (that was used as criterion for pair wise comparisons). The procedure is repeated upward for each level, until the top of the hierarchy is reached. The overall weight coefficient, with respect to goal for each decision alternative is then obtained. The alternative with the highest weight coefficient value should be taken as the best alternative. One of the major advantages of AHP is that it calculates the inconsistency index as a ratio of the decision maker's inconsistency and randomly generated index. This index is important for the decision maker to assure him that his judgments were consistent and that the final decision is made well. The inconsistency index should be lower than 0.10. Although a higher value of inconsistency index requires re- 
evaluation of pair wise comparisons, decisions obtained in certain cases could also be taken as the best alternative.

The elimination and choice translating reality (ELECTRE) method is capable of handling discrete criteria of both quantitative and qualitative in nature and provides complete ordering of the alternatives. The problem is to be so formulated that it chooses alternatives that are preferred over most of the criteria and that do not cause an unacceptable level of discontent for any of the criteria. The concordance, discordance indices and threshold values are used in this technique. Based on these indices, graphs for strong and weak relationships are developed. These graphs are used in an iterative procedure to obtain the ranking of alternatives (Nijkamp et al. 1990). This index is defined in the range (0-1), provides a judgment on degree of credibility of each outranking relation and represents a test to verify the performance of each alternative. The index of global concordance $C_{i k}$ represents the amount of evidence to support the concordance among all criteria, under the hypothesis that $A_{i}$ outranks $A_{k}$. It is defined as follow.

$$
C_{i k}=\sum_{j=1}^{m} w_{j} c_{j}\left(A_{i} A_{k}\right) / \sum_{j=1}^{m} w_{j}
$$

where $w_{j}$ is the weight associated with $j$ th criteria. Finally, the ELECTRE method yields a whole system of binary outranking relations between the alternatives. Because the system is not necessarily complete, the ELECTRE method is sometimes unable to identify the preferred alternative. It only produces a core of leading alternatives. This method has a clearer view of alternatives by eliminating less favorable ones, especially convenient while encountering a few criteria with a large number of alternatives in a decision making problem (Pohekar and Ramachandran, 2004, Shanian and Savadogo, 2006b).

All these methods allow cardinal data as input parameters, which reflects that the decision maker would use MADM methods only when a certain degree of information is available. Among the methods, there is no research shown that any one of them will perform better than others, but there is much dissension about the "truthfulness" of the results. (Steven 2000). This is because, under different assumptions and constraints, inconsistent rankings between two MADM methods are possible. Since the result from one method might not be enough to provide confidence to the decision maker, it is suggested that more than one method be applied. If ranking from different MADM methods are similar, the highest ranked alternative can be guaranteed to be the most preferable one to a decision maker as it has the highest confidence level.

However, the situation is not always optimistic. The worst case scenario might occur when a variety of ranked orders exist. In this case, MADM aggregation can be used to analyze them. In this step, all rankings from MADM methods are aggregated for finding a consistent result.

\section{MULTI-CRITERIA OPTIMIZATION METHODS}

There are different methods of multi-objective optimization being classified in terms of articulation of preferences in solving engineering problems. This includes the following:

* Methods with a priori articulation of preferences: This method allows the user or designer to specify preferences, which may be articulated in terms of relative importance of different objectives. Some of the methods that belong to this group are Linear aggregation/Weighted sum method, Compromise programming method, Weighted min-max method, Goal programming, Exponential weighted method, Weighted product method, Bounded objective function method, Lexicographic method, Reference Point Method, and desirability function approach, etc, (Marler et al. 2004; Mohammad 2005).

* Methods for a posteriori articulation of preferences: Sometimes, it is difficult for a decision maker to express an explicit approximation of the preference function. Therefore, it can be effective to allow the decision maker to choose from a range of solutions. The decision maker's preferences are presumably embedded in the parameter set. Multi-criteria optimizations that belong to this group are Physical programming method, Genetic algorithm, Normal boundary intersection (NBI) method and Normal constraint (NC) method.

- Methods with no articulation of preferences: Most times, the decision maker cannot actually define what he or she prefers. This group of multi-criteria methods does not require any articulation of preferences. Most of the methods are simplifications of the methods with a priori articulation of preferences mentioned above by setting all of the weights to one. They includes: Global criterion method, Compromise function, Objective sum, Min-max method, and Objective product

The general multi-objective optimization (MOO) problem is presented as follows:

$$
\underset{x}{\operatorname{Minimize}}: \mathrm{F}(\mathrm{x})=\left[F_{1}(x), F_{2}(x), \ldots, F_{k}(x)\right]^{T}
$$


Subject to: $g_{j}(x) \leq 0 ; \quad \mathrm{j}=1,2, \ldots, \mathrm{m}$

where $\mathrm{k}$ is the number of objective functions, $\mathrm{m}$ is the number of inequality constraints, $x \in E^{n}$ is a vector of design variables (also called decision variables), where $\mathrm{n}$ is the independent variables $x_{i}$, and $F(x) \in E^{k}$ is a vector of objective functions $F_{i}(x)$.

\subsection{The Weighted Sum Method}

The weighted sum model (or WSM) is probably the most commonly used approach, especially in single dimensional problems (Triantaphyllou et al. 1998). Using the weighted sum method to solve a problem entails selecting scalar weights $w_{i}$ and optimizing the following composite objective function:

$$
U=\sum_{i=1}^{k} w_{i} F_{i}(x)
$$

The weighting coefficient denotes the relative importance of the responses. Since a minimizing objective can be converted to a maximizing objective by multiplying it by -1 (i.e. minimize $f_{i}(x)=$ maximize $-f_{i}(x)$ ). Also the values of different functions or the coefficients of the terms in the functions may have different order of magnitude, it is necessary to normalize the objectives, in order to convert all objectives into the same dimensions or dimensionless before combining them into one. The objective functions may be converted to their normal forms as follows:

$$
F_{j}(x)=\left[\frac{w_{j}}{\sqrt{\sum_{j \in J} \beta_{i j}^{2}}}\right] f_{j}(x)
$$

for each $i \in I$ and $j \in J$

where $w_{j}$ is the weight assigned to objective $\mathrm{I}$, for each $i \in I$

$\beta_{i j}$ is the coefficient of term $\mathrm{j}$ in response function $\mathrm{i}$, for each $j \in J$ and $i \in I$

$f_{i}(x)$ is the objective function i, for each $i \in I$

Aggregate the objective functions into a single function as follows and add the structural constraints:

$$
\text { Maximize, } U=\sum_{i \in I}\left[\frac{\mathrm{w}_{\mathrm{i}}}{\sqrt{\sum_{\mathrm{j} \in \mathrm{J}} \beta_{\mathrm{ij}}^{2}}}\right] \mathrm{f}_{\mathrm{i}}(\mathrm{x})
$$

Subject to: $g_{j}(x) \leq 0 ; \quad j \in J$

where $g_{j}(x)$ is the feasible constraints.

\section{LIMITATION OF THE WEIGHTED-SUM METHOD}

In fact, a fundamental deficiency in the weighted sum method is that it can be difficult to discern between setting weights to compensate for differences in objective-function magnitudes and setting weights to indicate the relative importance of an objective as it is done with the rating methods. Since weighted sum approach involves a weight for each objective, it is sometimes difficult to achieve the task due to the objectives importance.

By combining all objectives in a single objective function (and transforming the multi-objective optimization problem in a single objective one) at one application of the algorithm can be obtained at most one solution. In order to obtain multiple solutions, the algorithm will be applied several times. Even then, you cannot be sure that all solutions are different. Running time required is another disadvantage of the weighted sum approach (Das and Dennis 1997).

Previous research has shown that the weighted sum method often produces poorly distributed solutions along a Pareto front, and that it does not find Pareto optimal solution in non-convex regions (Kim and de Weck, 2005; Messac et al. 2000a, 2000b). This means that an even distribution of the weights among objective functions does not always result in an even distribution of solutions on the Pareto front. 


\subsection{Compromise programming}

Compromise programming was first proposed by Zeleny (1973) and subsequently used by many researchers. This is a multi-criteria decision making (MCDM) technique, a powerful tool that can assist designers to select suitable material for a product design. The compromise programming identifies the best compromise solution as the one that has the shortest distance to an ideal point where the multiple objectives simultaneously reach their optimum values (Adeyeye and Oyawale, 2010). Compromise programming entails minimizing the difference between the potential optimal point and an ideal point (utopia point), $F^{*}=\left\{F_{1}{ }^{*}, F_{2}{ }^{*}, \ldots, F_{k}^{*}\right\}$, where $F_{\backslash j}{ }^{*}=\min _{x \in s} f_{j}(x)$. The ideal point is not practically achievable but may be used as a base point. The next best option is a solution that is as close as possible to the ideal point. Such a solution is called compromise solution and is Pareto optimal. The term close usually implies that one minimizes the Euclidean distance, $\left(L_{p}\right)$ which is defined as:

$$
L_{p}=\left|F_{j}(x)-F^{*}\right|=\left\{\sum_{i=1}^{k} w_{j}^{p}\left[F_{j}(x)-F_{j}^{*}\right]^{p}\right\}^{\frac{1}{p}}, \quad \mathrm{i}=1,2, \ldots, \mathrm{k}
$$

where $F_{j}(x)$ is the value of the objective functions,

For the $L_{1}$ metric $(\mathrm{p}=1)$, we achieve "the longest" distance geometrically speaking, the best compromise or closest solution to the ideal solution is obtained. The compromise programming method is illustrated with the data in example 1 with the same weight values (see table 5).

One of the limitation of the $\mathrm{CP}$ is that if $2 \leq \mathrm{p}<\infty$, then the objective function of the $\mathrm{CP}(\mathrm{p}, \mathrm{w})$ is nonlinear and not easy to handle. However, for $\mathrm{p}=\infty$, we have the $\mathrm{CP}(\infty, \mathrm{w})$ as a min-max problem.

To avoid biasing the distances computation, normalization is often required reducing unwanted effects resulting from the choice of measurement units or from the range of the domain. In maximization problems, this can be made with respect to the ideal point. However, in minimization problems, it is preferable to normalize using the nadir point. The nadir point is defined by the maximal values of each of the objectives within the set of Pareto optimal solutions, which is expressed as:

$$
F^{* * *}=\left\{F_{1}^{* * *}, F_{2}^{* *}, \ldots, F_{k}^{* *}\right\} \text {, where } F_{j}^{* * *}=\max _{x \in s} f_{j}(x) \text {. }
$$

Seeking a compromise is thus an effort to achieve an ideal solution and the quality of this compromise is then measured by its distance to the ideal solution. The relative importance assigned to each of the objectives by the decision-maker may also be expressed by a set of weights $w_{j} \geq 0$, and $\sum_{j=1}^{k} w_{j}=1$. Though the weights representing relative importance are used as the preference structure when applying $\mathrm{CP}$, it has been mathematically proven that $\mathrm{CP}$ is superior to the weighted-sum (WS) method in locating the efficient solutions, or the so called Pareto points (Steuer, 1986).

The general expression for calculating a Euclidean weighted and normalized distance $\left(L_{p}\right)$, between the values of the functions of the two solutions, $F_{j}(x)$ and $F_{j}^{*}$

$$
L_{p}=\left\{\sum_{j=1}^{k} w_{j}\left[\frac{F_{j}(x)-F_{j}^{*}}{F_{j}^{*}-F_{j}^{* *}}\right]^{p}\right\}^{\frac{1}{p}}
$$

Thus, the CP method allows one to seek solutions that approach the ideal point that is not itself generally feasible. The multiple objectives are thus represented within a single function that is the weighted and normalized distance to the ideal point.

\subsection{Weighted min-max method}

The weighted min-max formulation is given as follows:

$$
U=\max _{i}\left\{w_{i}\left[F_{i}(x)-F_{i}^{o}\right]\right\}
$$

A common approach for treating equation (10) is to introduce an additional unknown parameter $\lambda$ : 
$\underset{\mathrm{x} \in \mathrm{X}, \lambda}{\operatorname{Minimize}} \lambda$

subject to $\left.w_{i}\left\{F_{i}(x)-F_{i}^{o}\right\}\right\}-\lambda \leq 0, \mathrm{i}=1,2, \ldots, \mathrm{k}$.

$F_{i}(x)$ and $F_{i}^{o}$ are objective function and utopia point respectively.

However, increasing the number of constraints can increase the complexity of the problem.

The weighted min-max method is the limit of equation (7) as $p \rightarrow \infty$.

There are a lot of modifications made on equation (7) by several researchers, in order to alleviate the potential for solutions that are only weakly Pareto optimal. These modifications were seen in the work of Steuer and Choo, (1983); Kaliszewski (1985); Romero et al. (1998) using the augmented weighted Tchebycheff method and that of Kaliszewski, (1987) as shown in equation (12) and (13) respectively:

$$
\begin{aligned}
& U=\max _{i}\left\{w_{i}\left[F_{i}(x)-F_{i}^{o}\right]\right\}+\rho \sum_{j=1}^{k}\left[F_{j}(x)-F_{j}^{o}\right], \quad \mathrm{i}=1,2, \ldots, \mathrm{k} \\
& U=\max _{i}\left\{w_{i}\left[F_{i}(x)-F_{i}^{o}+\rho \sum_{j=1}^{k}\left(F_{j}(x)-F_{j}^{o}\right)\right]\right\}, \quad \mathrm{i}=1,2, \ldots, \mathrm{k}
\end{aligned}
$$

where $\rho$ is a sufficiently small positive scalar assigned by the decision maker. Steuer (1989) suggests that (13) is necessary and sufficient for Pareto optimality as long as $\rho$ is not too large. $d_{m}$

Application of this method is illustrated with data in table 1 and 2 as shown in table 6.

Using equation (10), we have $U=\max _{i}\left\{w_{i}\left[F_{i}(x)-F_{i}{ }^{o}\right]\right\}$

where $\mathrm{U}$ is the utility function, $F_{i}^{o}=\min _{x}\left\{f_{i}(x) \mid x \in X\right\}$, as the utopia point or ideal point, the $w_{i}$ is a vector of weights typically set by the decision maker such that $\sum_{i=1}^{n} w_{i}=1$ and $\mathrm{w}>1$.

\subsection{Weighted product method (WPM)}

The weighted product model (or WPM) is very similar to the WSM. The main difference is that instead of addition in the model there is multiplication. Each alternative is compared with the others by multiplying a number of ratios, one for each criterion. Each ratio is raised to the power equivalent to the relative weight of the corresponding criterion.

The Weighted Product Method was introduced by Bridgeman (1922) and was referring to as product of powers. Other researchers like Gerasimor and Repko (1978) have successfully apply the method to minimize the weight, displacement, and difficulty of construction, where the cross-sectional areas of the rods are the design variables, and constraints are strength and stability. However, the approach has not been used extensively, and the characteristics of the weights are unclear. This lack of extensive use could be the result of potential nonlinearities in the utility function and consequent computational difficulties.

Contrary to the weighted-sum method, the different measurement units here do not have to be transformed into a dimensionless scale by a normalization process. This is because in the WPM method, the attributes are connected by multiplication. The weights become exponents associated with each criteria value (positive power for beneficial criteria, and negative power for non-beneficial criteria). The multi-criteria utility function $U$ of alternative $X_{i}$ is given by:

$U=\prod_{i=1}^{n}\left[F_{i}(x)\right]^{w_{i}}$

where $w_{i}$ is the weights indicating the relative importance of the objective functions.

\subsection{Desirability function approach}

The concept of desirability was first introduced by Harrington (1965), as a method for multi-criteria optimization. The approach was further modified by Derringer and Suich (1980) and Kim and Lin (2000). In desirability function approach, each desirability $\left(d_{i}\right)$ function may be related to particular design objectives, $f_{i}(x)$. Considering the simultaneous effects of multiple objectives or individual objective functions, 
a systematic transformation scheme (from $f_{i}(x)$ to $d_{i}$ ) can be utilized to aggregate every value of individual desirability into a single global desirability index through a weighted geometric mean (Yetilmezsoy 2012).

In order to discover the global optimal conditions, each predicted $i$ th objective $f_{i}(x),(i=1,2, \ldots, \mathrm{n})$ is transformed to a scale free desirability value $d_{i}\left(0 \leq d_{i} \leq 1\right)$, where $d_{i}=0$ represents completely undesirable objective and $d_{i}=1$ represents completely desirable or ideal response. Generally, the goal of optimization is to maximize desirable objectives, minimize undesirable objectives, and hit the target level of some. If the objective function is to be maximized (Larger-the-Better (LTB) type), its individual desirability function $d_{i}$ is defined as:

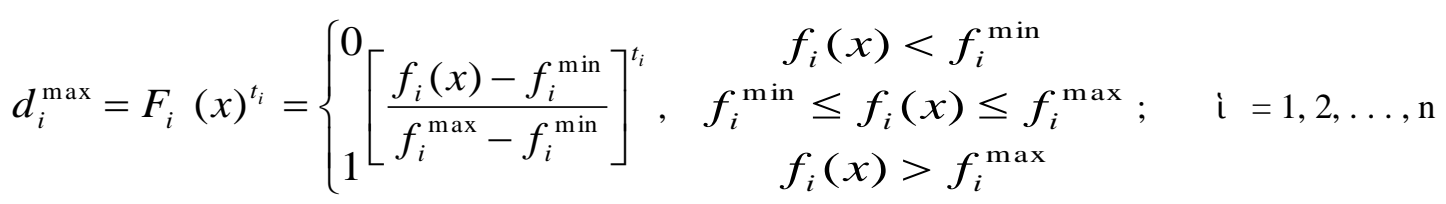

where $\mathrm{n}$ denotes the number of objectives, $f_{i}^{\mathrm{min}}$ and $f_{i}^{\mathrm{max}}$ are the minimum and maximum acceptable values of $f_{i}(x)$ respectively, $t_{i}$ is an exponent chosen to reflect how rapidly the deviation from the target value of objective í becomes undesirable, for each $i \in I$. If the objective function of interest is minimization (smallerthe-better (STB) type), the individual desirability function $d_{i}$ is defined as follows:

$$
d_{i}^{\min }=F_{i}(x)^{w_{i}}=\left\{\begin{array}{c}
1 \\
{\left[\begin{array}{l}
f_{i}(x)-f_{i}^{\max } \\
f_{i}^{\text {min }}-f_{i}^{\max }
\end{array}\right]^{t_{i}} ; f_{i}^{\text {min }} \leq f_{i}(x) \leq f_{i}^{\text {max }} \text { where } w_{i}>0} \\
f_{i}(x)>f_{i}^{\text {max }}
\end{array}\right.
$$

And for target is best situation, the desirability function becomes:

$$
d_{i}^{\text {target }}=F_{i}(x)^{w_{i}}=\left\{\begin{array}{lc}
{\left[\frac{f_{i}(x)-f_{i}^{\text {min }}}{T_{i}-f_{i}^{\text {min }}}\right]^{t_{i}}} & f_{i}^{\text {min }} \leq f_{i}(x)<T_{i} \\
{\left[\frac{f_{i}(x)-f_{i}^{\text {max }}}{T_{i}-f_{i}^{\text {max }}}\right]^{\gamma_{i}}} & T_{i} \leq f_{i}(x) \leq f_{i}^{\text {max }} \\
0, & \text { otherwise }
\end{array}\right.
$$

where $\gamma_{i}$ is an exponent chosen to reflect how rapidly the deviation from the target value of response í towards its lower limit becomes undesirable, for each $i \in I$

The values of $t_{i}$ and $\gamma_{i}$ can be chosen so that the desirability criterion is easier or more difficult to satisfy. Consequently, the desirability approach can convert a multi-objective problem into a single-objective one. Then, the optimal conditions for several objectives is obtained by maximizing the overall (global) desirability $\mathrm{D}$, which is the weighted geometric mean of all the individual desirability functions $(0 \leq D \leq 1)$ (Coffey et al. 2007; Akalin et al. 2010), given as:

$D=\left[\prod_{i=1}^{n} d_{i}^{w_{i}}\right]^{1 / \sum_{i=1}^{n} w_{i}}$

where $\mathrm{D}$ is the overall desirability, $\mathrm{n}$ is the number of objectives, $w_{i}$ is the relative importance of the $\mathrm{i}$ th objective and $\sum_{i=1}^{n} w_{i}=1$.

\subsection{The Reference Point Method}


A paper presented by Kalyanmoy et al. (2006) also discussed on multi-objective optimization based on reference point and using evolutionary algorithms. In their paper, the concepts of reference point methodology in an evolutionary multi-objective optimization was used and attempt to find a set of preferred Pareto optimal solutions near the regions of interest to a decision maker. The procedure adopted is equivalent to using a weight vector emphasizing each objective function equally or using $w_{i}=\frac{1}{m}$. This means that if the decision-maker is interested in biasing some objective more than others, a suitable weight vector can be used with each reference point and instead of emphasizing solutions with the shortest Euclidean distance from the reference point. The Euclidean distance measure with the following weighted Euclidean distance measure

$$
d_{i j}=\sqrt{\sum w_{i}\left(\frac{f_{i}(x)-\bar{Z}}{f_{i}^{\text {max }}-f_{i}^{\text {min }}}\right)^{2}}
$$

where $f_{i}^{\max }$ and $f_{i}^{\text {min }}$ are the population maximum and minimum function values of $i^{\text {th }}$ objective. The weighted distance measure can also be used to find a set of preferred solutions in the case of problems having non-convex Pareto-optimal front, where $w_{i}$ is the $i^{\text {th }}$ component of a chosen weight vector used for scalarizing the objectives.

$Z$ reference point for an $\mathbf{M}$ - objective optimization problem of minimizing $f_{1}(x), \ldots, f_{m}(x)$ with $x \in s$.

The authors proposed interactive evolutionary multi-objective optimization (EMO) and decisionMaking using Reference direction method that borrows the concept of reference direction approach from the multi-criterion decision-making literature and combine with an evolutionary multi-objective optimization procedure to develop an algorithm for finding a single preferred solution in a multi-objective optimization scenario efficiently.

EMO methodologies have demonstrated to find a set of trade-off optimal solutions in solving multiobjective optimization problems. The interactive EMO methods can hope to achieve the following tasks by exploiting the population aspect of EMO.

1. Instead of finding a single preferred solution on the Pareto-optimal front, an EMO can assist in finding a set of preferred solution or a preferred region on the Pareto-optimal set. The advantage of finding a region of solutions instead of a single solution is that (i) the decision-maker can provide a tentative information about his/her preference (i.e., the reference point need not be precisely chosen), (ii) a set of solutions near a preferred solution provides information about other solutions which are close to the preferred solution but may have interesting trade-off for the decision-maker to consider and (iii) the knowledge of more than one solution near the preferred point may help decipher common properties of such solutions, thereby providing salient information about desired solutions.

2. Instead of finding preferred solutions near a single portion of the Pareto-optional frontier, an EMO can help find multiple preferred regions corresponding to differing preferences simultaneously. This task is particularly useful if the decision- maker is not sure whether to concentrate near a single preferred region or to explore multiple preferred regions simultaneously.

3. EMO can replace any repetitive application of single objective optimizations which may be needed in a classical interactive multi-objective optimization and decision-making task.

Some of the limitations of this method are as follows (Janusz and Marek, 2006):

- Preferences are specified for each criterion separately; this supports an easy and precise definition of preferences but less experienced users may have problems with correct interpretation of such preferences in terms of trade-offs between criteria.

- Specification of preferences requires more information than required for the weighted criteria approach.

- Modification of preferences aimed at examining certain regions of Pareto sets might be difficult for problems with many criteria.

- Qualitative criteria must be mapped into a quantitative scale

The known implementations do not deals with hierarchical criteria structures.

\subsection{Physical Programming}

Initially developed by Messac (1996), physical programming maps general classifications of goals and objectives, and verbally expressed preferences to a utility function. It provides a means of incorporating preferences without having to conjure relative weights. Objective functions, constraints, and goals are treated equivalently as design metrics. In general, the decision maker customizes an individual utility function, which is 
called a class function $\bar{F}_{i}\left[F_{i}(x)\right]$, for each design metric. Specifically, each type of design metric is first associated with a type of individual utility function distinguished by a general form, such as a monotonically increasing, monotonically decreasing, or unimodal function. Then, for each metric, the decision-maker specifies the numerical ranges that correspond to different degrees of preference (desirable, tolerable, undesirable, etc.). These ranges include limits on the values of the metrics, which are modeled as additional constraints. Because of the way these class functions are constructed, physical programming is able to effectively optimize objective functions with significantly different orders of magnitude (Messac et al. 2004). The requirement that the decision maker quantitatively classify different ranges of values for each metric can be viewed in two ways. On one hand, it suggests that physical programming requires significant familiarity with each objective and constraint. On the other hand, in a more positive light, it implies that physical programming allows one to make effective use of available information. The individual utility functions, as non-dimensional unimodal transformations, are combined into a utility function as follows:

$F_{\alpha}(x)=\log \left\{\frac{1}{d_{m}} \sum_{i=1}^{d_{m}} \bar{F}_{i}\left[F_{i}(x)\right]\right\}$

where $\mathrm{dm}$ represents the number of design metrics being considered.

$\bar{F}_{i}$ is the mean value of the response for each $i \in I$

Messac et al. (2001) prove that physical programming provides a sufficient condition for Pareto optimality. In addition, Messac and Mattson (2002) demonstrate how physical programming can be used as a necessary condition for Pareto optimality, providing all Pareto optimal points. In fact, it is superior to the weighted sum method and to compromise programming in its ability to represent the complete Pareto optimal set with an even distribution of points (Chen et al. 2000; Messac 2000a; Messac et al. 2001).

\subsection{The Goal programming Method}

Goal programming has become one of the most powerful and popular theoretical method for dealing with multi-objective programming techniques (Hokey and James, 1991). Charnes et al. (1955), Charnes and Cooper (1961), Ijiri (1965), Charnes et al. (1967) and many others has been instrumental in the development of various forms of goal programming method. Actually, Charnes and Cooper (1961) first introduced the idea of 'Archimedean' goal programming method, in which goals $g_{j}$ are specified for each objective function $F_{j}(x)$. Then the total deviation from the goals $\sum_{j=1}^{k}\left|d_{j}\right|$ is minimized, where $d_{j}$ is the deviation from the goal $g_{j}$ for the jth objective. To model the absolute values, $d_{j}$ is split into positive and negative parts such that $d_{j}=d_{j}^{+}-d_{j}^{-}$, with $d_{j}^{+} \geq 0, d_{j}^{-} \geq 0$, and $d_{j}^{+} d_{j}^{-}=0$. Also, $\left|d_{j}\right|=d_{j}^{+}+d_{j}^{-}$, where $d_{j}^{+}$and $d_{j}^{-}$ represent overachievement and underachievement respectively. Archimedean goal programming (or weighted goal programming constitutes a subclass of goal programming, in which weights are assigned to the deviation of each objective from its perspective goal (Charnes and Cooper 1977). In GP model, all of the objectives are assigned target levels for achievement and a relative importance for achieving these levels. GP treats these targets as goals to aspire to and not as absolute constraints. Goal programming searches for a way to come as close as possible to reaching the goals, the objective of this technique is to minimize the sum of the deviations for all the goals (Chen and Shyu, 2006). There are many applications of goal programming, such as weapon selection system, supplier selection problem, production planning, for finding efficient asset-liability management of property-liability insurer, and many more.

There are two cases of goal programming, namely; (i) Non preemptive Goal Programming (NGP), (ii) Pre-emptive Goal programming (PGP). In the first type it is not possible to achieve all the goals because of their conflicting nature, and due to the fact that all goals are of roughly comparable importance, and there will be deviations from their target values for all or some of the responses. These unwanted deviations are assigned weights according to their relative importance to the decision maker (DM) and should be minimized as an Archimedean sum (Tamiz et al. 1998). This is known as weighted GP (WGP). (The preemptive (or lexicographic) goal programming approach is similar to the lexicographic method in that the deviations $\left|d_{j}\right|=d_{j}^{+}+d_{j}^{-}$for the objectives are ordered in terms of priority and minimized lexicographically. A lexicographic minimization may be defined as a sequential minimization of each priority while maintaining the minimal value. There is a hierarchy of priority levels for the objective function, so that primary importance 
receive first priority attention, those of secondary importance receive second priority attention and so forth (Adeyeye and Oyawale, 2010).

GP approaches have been applied to solve many real-world problems. However, many multiple-choice aspiration levels may exist, such as "something more/higher is better" or "something less/lower is better" (Chang, 2007). These typical multiple choice GP problems cannot be solved using the traditional GP approach. Chang (2007) presented a multi-criteria goal programming (MCGP) method to solve these types of problems, and it can be expressed as follows:

(MCGP model)

$$
\min \sum_{j=1}^{n}\left(\alpha_{j} d_{j}^{+}+\beta_{j} d_{j}^{-}\right)
$$

Subject to $f_{j}(x)-d_{j}^{+}+d_{j}^{-}=\mathrm{g}_{\mathrm{j} 1}$ or $\mathrm{g}_{\mathrm{j} 2} \ldots$ or $\mathrm{g}_{\mathrm{jm}}$

$$
\begin{aligned}
& d_{j}^{+}, d_{j}^{-} \geq 0, j=1,2, \ldots, n \\
& x \in F(\mathrm{~F} \text { is a feasible set), }
\end{aligned}
$$

where $g_{j i}(\mathbf{i}=1,2, \ldots, \mathrm{m}$ and $\mathrm{j}=1,2, \ldots, \mathrm{n})$ is the íth aspiration level of the $\mathrm{jth}$ goal;

$\alpha_{j}$ and $\beta_{j}$ are the weights reflecting preferential and normalizing purposes attached to positive and negative deviations of jth goal, respectively; $d_{j}^{-}=\max \left(0, g_{j}-f_{j}(x)\right)$ and $d_{j}^{+}=\max \left(0, f_{j}(x)-g_{j}\right)$ are, respectively, under- and over-achievements of the jth goal, $f_{j}(x)$ is the linear function of the jth goal, and $g_{j}$ is the aspiration level of the jth goal.

\section{Disadvantages of Goal programming}

- GP requires that the decision maker specify fairly detailed a priori information about his or her aspiration levels, preemptive priorities, and the importance of goals in the form of weights.

- In many complex problems, it is difficult (or even impossible) for the decision maker to provide the precise information required by these methods

NOTE: studies have shown that decision makers find it relatively easy to specify ordinal rankings for goals, but they are unable to derive meaningful preference weights on a cardinal scale. These difficulties are aggravated further when the goals are not related to each other.

\section{DISCUSSION}

The Weighted Sum Method is suitable for situations when the designer is interested in minimizing undesirable objective functions while at the same time, also wants to maximize desirable responses.

The Compromise Programming is useful when one wants to identify the closest distance to an ideal solution, where the multiple objectives simultaneously reach their optimum point and idea point. Its effectiveness depends on the value of the exponent $p$.

The Weighted Product Method is applied mostly when the designer allows functions with different orders of magnitude to have similar significance and also to avoid objective function transformation. However, the method lacks extensive use as a result of potential nonlinearities in the utility function and consequent computational difficulties.

The weighted min-max method is equally suitable approach that can provide the complete Pareto optimal set with variation in the weights; it also provides a necessary condition for Pareto optimality. In addition, it is sufficient for weak Pareto optimality.

Desirability function approach is suitable when the designer maker wants to minimize some objective functions, maximize some and achieve target values for some objective functions simultaneously.

The reference point method is applied mostly in an evolutionary multi-objective optimization in finding a single preferred solution in a multi-objective optimization, and the procedure allows the decision maker in biasing some objective more than others using a suitable weight vector with each reference point.

Physical programming is an effective method in providing Pareto optimal points that accurately represent the complete Pareto optimal set. It provides a means of incorporating preference without having to conjure relative weights. This means that physical programming operates based on imposed preferences; it provides a means to circumvent the use of weights, which may be awkward. Here, the constraints and objectives may be treated equivalently as design metrics. Moreover, the programming complexity is high. 
The goal programming is generally suitable for cases where the designer wants to achieve target values for the objective functions and the functions are of comparable importance. In addition, it is useful when the objective functions are in hierarchical order of importance, especially when designer wants to achieve lower priority objective(s) without sacrificing the achievement of higher priority at the same time.

Given the variety of methods discussed in this paper, the question arises as to which method is the best. Unfortunately, there is no distinct answer. However, methods that provide both necessary and sufficient conditions for Pareto optimality are preferable.

\section{CONCLUSION}

In general, multi-criteria optimization requires more computational effort than single-criteria optimization. Methods with a priori articulation of preferences require the user to specify preferences only in terms of objective functions. Alternatively, methods with a posteriori articulation of preferences allow the user to view potential solutions in the criterion space.

Selection of a specific scalarization method for a priori articulation of preferences which allows the user to design a utility function depending on the type of preferences that the decision maker wishes to articulate.

Most multi-criteria optimization algorithms depend on the efficiency of the single-objective optimization algorithm. Hence, it is necessary to select an efficient single-objective optimization algorithm and associated software.

Moreover, methods with a posteriori articulation of preference are less efficient than methods with a priori articulation of preferences in terms of computer process unit time.

Though, this paper has not exhausted the multi-criteria optimization methods. Many other multi-criteria methods such as reference point method, genetic algorithm, exponential weighted, bounded objective function, etc. may also be useful.

\section{REFERENCES}

[1] Adeyeye A. D. and Oyawale A. F. (2010). Multi-objective methods for welding flux performance optimization. RMZ-Materials and Geoenvironment 57.2: 251-270.

[2] Akalin, O., Akay, K. U., Sennaroglu, B., Tez, M. (2010). Optimization of chemical admixture for concrete on mortar performance tests using mixture experiments. Chemom. Intelligence Laboratory System. vol 104, pp. 233-242.

[3] Ashby M. F. (2000). Multi-objective optimization in material selection. Acta Materilia, vol. 48, pg. 359369.

[4] Ashby M. F. (2005). Materials selection in mechanical design, Third Edition, Elsevier.

[5] Bridgman, P. W. (1922). Dimensional Analysis. New Haven. Yale University Press.

[6] Chang, C. T. (2007). Multi-choice goal programming. Omega, vol. 35, No. 4. Pp. 389-396.

[7] Chang, C. T. (2007). Multi-choice goal programming. Omega, vol. 35, No. 4. Pp. 389-396.

[8] Charnes, A., Clower, R.W., Kortanek, K.O. (1967). Effective control through coherent decentralization with preemptive goals. Econometrica 35, 294-320.

[9] Charnes, A., Cooper, W. W. (1961). Management Models and Industrial Applications of Linear Programming. New York: John Wiley and Sons. 391.

[10] Charnes, A., Cooper, W. W. (1977). Goal programming and multiple objective optimization; Part 1. European Journal of Operational Research vol. 1. Pg. 39-54.

[11] Charnes, A., Cooper, W.W., Ferguson, R.O. (1955). Optimal estimation of executive compensation by linear programming. Management Science. 1, 138-151.

[12] Chen, R., Shyu, Z. J. (2006). Selecting a weapon system using zero-one goal programming and analytic network process. Journal of Information \& Optimization Sciences, vol. 27 (2), No. 2, pp. 379-399.

[13] Chen,W.; Sahai, A.; Messac, A.; Sundararaj, G. (2000): Exploration of the effectiveness of physical programming in robust design. J. Mech. Des. 122, 155-163.

[14] Chi-Nung, L., Hsing-Pei, K. (2011). An integrated fuzzy TOPSIS and MCGP approach to supplier selection in supply chain management. Expert Systems with Appliaction, vol. 38, pp. 10803-10811.

[15] Das, I., Dennis, J. E., (1997). A closer look at drawbacks of minimizing weighted sums of objectives for Pareto set generation in multi-criteria optimization problems. Structural Optimization. 14, 63-69.

[16] Deb, K. (2001). Nonlinear Goal programming using Multi-objective Genetic Algorithms. Journal of the Operational Research Society, vol. 52, No. 3, Pg. 291- 302.

[17] Derringer, G. C., and Suich, D. (1980): Simultaneous optimization of several response variables; Journal of Quality Technology 12 (4), pp. 214-219. 
[18] Elahi B., Seyed-Hosseini S. M., \& Makui A. (2011). A Fuzzy Compromise Programming Solution for Supplier Selection in Quantity Discounts Situation. International Journal of Industrial Engineering and production Research, Vol. 22, No. 2, Pg. 107-114.

[19] Figueira J, Greco S, Ehrgott M, editors. (2005). Multiple criteria decision analysis: State of the art surveys. New York (NY): Springer.

[20] Gerasimor, E. N., Repko, V. N. (1978). Multi-criteria Optimization. Journal of Applied Mechanics, vol 14, pg. 1179-1184.

[21] Hokey M. and James S., (1991). On the origin and persistence of misconceptions in goal programming. Journal of Operational Research Society. Vol. 42, No. 4. Pp. 301-312.

[22] Ijiri, Y. (1965). Management Goals and Accounting for Control. Amsterdam: North-Holland.

[23] Janusz G., Marek M., (2006). Multi-criteria methodology for the NEEDs project. Integrated Project: New Energy Externalities Developments for Sustainability (NEEDs).

[24] Kaliszewski, I. (1985). A characterization of properly efficient solutions by an augmented Tchebycheff norm. Bull. Pol. Academic Science and Technological Science. 33, 415-420.

[25] Kaliszewski, I. (1987). A modified Tchebycheff metric for multiple objective programming. Computational Operation Research. 14, 315-323.

[26] Kalyanmoy Deh, Sundar J., Udaya Bhaskara Roa N., and Shamik Chaudhuri, (2006). Reference Point based Multi-objective Optimization using Evolutionary Algorithms. International Journal of Computational Intelligence Research. ISSN 0973-1873 vol. 2, No. 3, pp. 273-286.

[27] Kim I. Y., de Weck O. L., (2005). Adaptive weighted sum method for bi-objective optimization: Pareto front generation. Structural Multidisciplinary Optimization. Vol. 29, pg. 149-158. DOI: 10.1007/s00158004-0465-1.

[28] Kim, K. J., and Lin, J. (2000): Simultaneous optimization of mechanical properties of steel by maximizing desirability functions; Applied Statistics 49 (3), pp. 311-326.

[29] Koksalan M., Wallenius J., Zionts S., (2013). An Early History of Multiple Criteria Decision Making. Journal of Multi-Criteria Decision Analysis. Vol. 20, Pg. 87-94.

[30] Koski, J. (1980). Truss Optimization with Vector Criterion, Examples. Report Number 7, Tampere University of Technology, Tampere, Finland.

[31] Koski, J. 1979: Truss Optimization with Vector Criterion. Report Number 6, Tampere University of Technology, Tampere, Finland.

[32] Linkov, I., Varghese, A., Jamil, S., Seager, T.P., Kiker, G. and Bridges, T. (2004). Multi-criteria decision analysis: A framework for structuring remedial decisions at the contaminated sites., In: Linkov, I. and Ramadan, A.B. (Eds.). Comparative Risk Assessment and Environmental Decision Making, Springer, New York, pp. 15-54.

[33] Marler R. T., Arora J. S. (2004). Survey of multi-objective optimization methods for engineering. Struct. Multidisciplinary Optimization 26, 369-395.

[34] Marler R. T., Arora J. S. (2009). The weighted-sum method for multi-objective optimization: new insights. Structural Multidisciplinary Optimization. DOI 10.1007/s00158-009-0460-7

[35] Messac, A. (1996): Physical programming: effective optimization for computational design. AIAA J. 34, $149-158$.

[36] Messac, A. (2000): From dubious construction of objective functions to the application of physical programming. AIAA J. 38, 155-163.

[37] Messac, A., Sukam, C. P., Melachrinoudis, E. (2000a): Aggregate objective functions and Pareto frontiers: required relationships and practical implications. Journal of Optimization Eng. 1, 171-188.

[38] Messac, A., Sundararaj, G.J., Tappeta, R.V., Renaud, J. E. (2000b): Ability of objective functions to generate points on nonconvex Pareto frontiers. AIAA J. 38, 1084-1091.

[39] Messac, A.; Hattis, P. (1996): Physical programming design optimization for high speed civil transport (HSCT). J. Aircr. 33, 446-449.

[40] Messac, A.; Mattson, C.A. (2002): Generating well-distributed sets of Pareto points for engineering design using physical programming. Optim. Eng. 3 431-450.

[41] Messac, A.; Puemi-Sukam, C.; Melachrinoudis, E. (2001): Mathematical and pragmatic perspectives of physical programming. AIAA J. 39, 885-893.

[42] Messac, A.; Wilson, B. (1998): Physical programming for computational control. AIAA J. 36, 219-226.

[43] Mohammad H. K. (2005). Robust Multi-criteria Optimization of Surface Location Error and Material Removal Rate in High-Speed Milling under uncertainty. A Dissertation presented to the Graduate School of the University of Florida in Partial Fulfillment of the Requirement for the Degree of Doctor of Philosophy, University of Florida.

[44] Nijkamp P., Rietveld P., and Voogd H. (1990). Multicriteria Evaluation in Physical Planning, NorthHolland, Amsterdam. 
[45] Pohekar S. D., Ramachandran M. (2004). Application of Multi-criteria decision making to sustainable energy planning - A review. Renewable and Sustainable Energy Reviews. 8:365-381.

[46] Rao R. V., Patel B. K., (2011). Material Selection Using a Novel Multiple Attribute Decision Making Method. International Journal of Manufacturing Materials and Mechanical Engineering, Vol. 1, No. 1, Page 43-56.

[47] Rao R.V. and Davin J. P. (2008). A decision-making framework model for material selection using a combined multiple attributes decision-making method. International Journal of advance Manufacturing Technology; vol. 35; pg. 751-60.

[48] Romero, C. (1985). Multi-objective and Goal programming Approaches as a Distance Function Model. Journal of the Operational Research Society, vol. 36, N0. 3, Pg. 249-251.

[49] Romero, C., Amador, F., Barco, A. (1987). Multiple Objectives in Agricultural Planning: A Compromise Programming Application. American Journal of Agricultural Economics, Vol. 69, No. 1, Pg. 78-86.

[50] Romero, C.; Tamiz, M.; Jones, D.F. (1998). Goal programming, compromise programming and reference point method formulations: linkages and utility interpretations. Journal of Operation Research Society. 49, 986-991.

[51] Shanian A., Savadogo O. (2006a). A non-compensatory compromised solution for material of bipolar plats for polymer electrolyte membrane fuel cell. Journal of Electrochimica. Acta 51 pp. 5307-5315.

[52] Shanian A., Savadogo O., (2006b). A Material selection model based on the concept of multiple attribute decision making. ScienceDirect: Materials \& Design, vol. 27, issue 4. Doi: 10.1016/j.matdes.2004.10.027.

[53] Shanian A., Savadogo O., (2009). A methodological concept for material selection of highly sensitive components based on multiple criteria decision analysis. Expert Systems with Application. An International Journal Archive, volume 36, Issue 2, pg. 1362-1370 Part 1, Published by Pergamon Press, Inc. Tarrytown, NY, USA.

[54] Stadler W. (1984). A Survey of Multi-optimization or the Vector Maximum Problem, Part I: 1776-1960. Journal of Optimization Theory and Applications. vol. 29, No. 1, pp. 1-52.

[55] Steuer, R.E. (1989). Multiple Criteria Optimization: Theory, Computation, and Application. Malabar: Robert E. Krie- ger Publishing.

[56] Steuer, R.E.; Choo, E.-U. (1983). An interactive weighted Tchebycheff procedure for multiple objective programming. Mathematical Programming. 26, 326-344.

[57] Steven K. C. (2000). Development of a Fuzzy Multi-Criteria Decision Support System for Municipal Solid Waste Management. Unpublished Thesis Submitted to the Faculty of Graduate Studies and Research University of Regina, Saskatchewan.

[58] Tamiz, M., Jones, D., Romero, C. (1998). Goal programming for decision making: An overview of the current state-of-the art. European Journal of Operational Research, vol. 111, 569-581.

[59] Triantaphyllou, B., Shu, S., Nieto Sanchez, and Ray T. (1998). Multi-criteria Decision Making: An Operations Research Approach. Published in Encyclopedia of Electrical and Electronics Engineering, (J. G. Webster, Ed), John Wiley \& Sons, New York, NY, Vol. 15, Pg. 175-186.

[60] Yetilmezsoy, K. (2012). Integration of kinetic modeling and desirability function approach for multiobjective optimization of UASB reactor treating poultry manure wastewater. Journal of Bioresource Technology, vol. 118, pp. 89-101.

[61] Yoe C. (2002). Trade-off Analysis Planning and Procedures Guidebook. Prepared for Institute for Water Resources. U. S. Army Corps of Engineers.

[62] Zeleny, M. (1973). Compromising programming, Multiple criteria decision making, University of South Carolina Press, Columbia, USA.

[63] Zimmermann, H. J., (1991) Fuzzy Set Theory and Its Applications, Kluwer Academic Publishers, Second Edition, Boston, MA. 